\title{
Optimizing the ink formulation for preparation of Cu-based Gas Diffusion Electrodes yielding ethylene in electroreduction of $\mathrm{CO}_{2}$
}

\author{
Liniker de Sousal,2, Christian Harmokol, Nieck Benes ${ }^{2}$, Guido Mul ${ }^{\text {I* }}$ \\ ${ }^{1}$ Photocatalytic Synthesis Group, Faculty of Science and Technology, MESA+ Institute for \\ Nanotechnology, University of Twente, P.O. Box 217, 7500 AE Enschede, The Netherlands. \\ ${ }^{2}$ Membrane Science and Technology Cluster, Faculty of Science and Technology, MESA+ \\ Institute for Nanotechnology, University of Twente, P.O. Box 217, 7500 AE Enschede, The
}

Netherlands.

*E-mail: g.mul@utwente.nl 
S1: Schematic view of copper-based gas diffusion electrodes preparation.

Figure S1 presents the preparation method of copper-based GDEs through ink-coating using an air-brush. The carbon substrate was first cut in a piece of $2 \mathrm{~cm} \times 3 \mathrm{~cm}$ and coated onto an effective area of $1 \mathrm{~cm} \times 2 \mathrm{~cm}$, resulting in a $2 \mathrm{~cm}^{2}$ geometric area. All catalysts were dried under the same temperature $-160^{\circ} \mathrm{C}-$ for $16 \mathrm{~h}$.
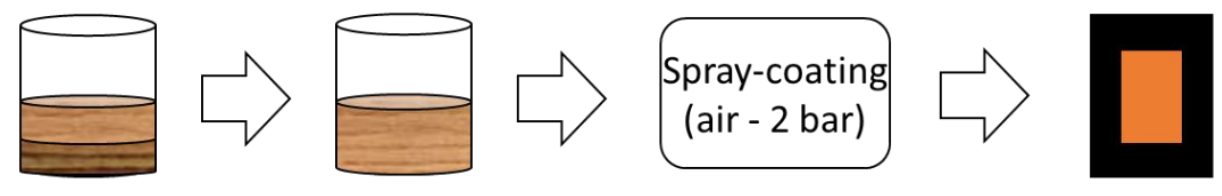

$$
2 \mathrm{~cm}^{2}
$$


Figure S1. Cu-GDE catalysts preparation procedure.

S2: Electrochemical catalytic surface area (ECSA) determination by double-layer capacitance (DLC) method for Cu-GDE sample prepared using isopropanol (IPA) as ink-solvent.

Figure S2 shows cyclic voltammograms obtained for the catalyst $\mathrm{Cu}$-GDE prepared using IPA as ink solvent. Varying the CV scan rate and using the cathodic half-way I-V charge, we could calculate the double-layer capacitance as the slope of the curve determined by current-density-scan rate graph. 


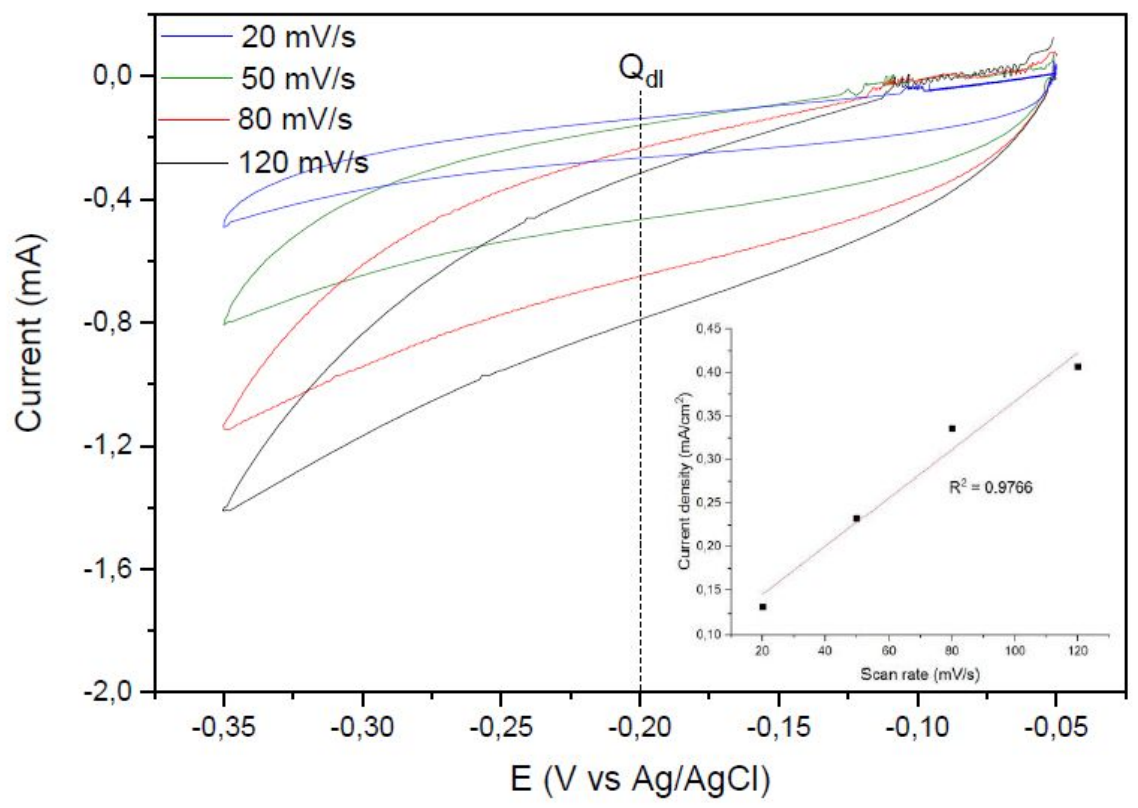

Figure S2. Double layer capacitance measurements for Cu-GDE sample prepared using IPA as dispersion solvent.

S3: Electrochemical impedance spectroscopy (EIS) and equivalent circuit description

Figure S3 depicts the equivalent circuit used to simulate and calculate ohmic cell resistance $\left(\mathrm{R}_{\text {cell }}\right)$ and charge-transfer resistance $\left(\mathrm{R}_{\mathrm{ct}}\right)$ of Cu-GDE samples. Open circuit voltage $(\mathrm{OCV})$ has 
been first measured for 5 mins to ensure the stability of the reactor followed by potentioelectrochemical impedance spectroscopy (PEIS) at $0 \mathrm{~V}$ vs OCV to evaluate the polarization/corrosion resistance at OCV and at $-0.94 \mathrm{~V}$ vs RHE to determine $\mathrm{R}_{\mathrm{o}}$ and $\mathrm{R}_{\mathrm{ct}}$. The measurements were performed in a homemade continuous flow-cell, in a frequency range of $10 \mathrm{kHz}$ to $100 \mathrm{mHz}$ using $10 \mathrm{mV}$ of amplitude and single sine wave mode. The measured data was fitted using Boukamp model and simulated by Randles-equivalent circuit without Warburg element, in which the capacitance is replaced by a constant-phase element for a better fit and simulating a porous environment. During the fitting process, all constant phase element (CPE) factor obtained were $>0.8$ which is essential to ensure that the capacitance is close to ideality.

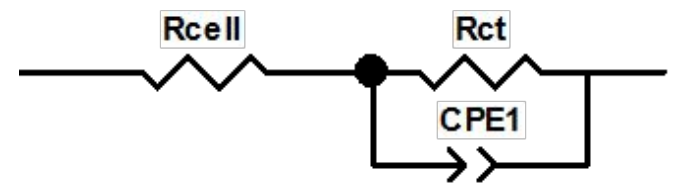

Figure S3. Illustration of the circuit elements used for data simulation and resistance calculation. 
S4: X-ray diffractograms of $\mathrm{Cu}-\mathrm{GDE}$ samples prepared using different ink-dispersion solvents.

All configurations have presented similar pattern (no broadening) in copper region and same orientation (111), (200) and (220), even after drying procedure. The three main peaks were associated with PDF 04-0836 assigned to metallic copper phase. XRD of bare GDL and bare copper powder can be found in Figure S4 as well. 


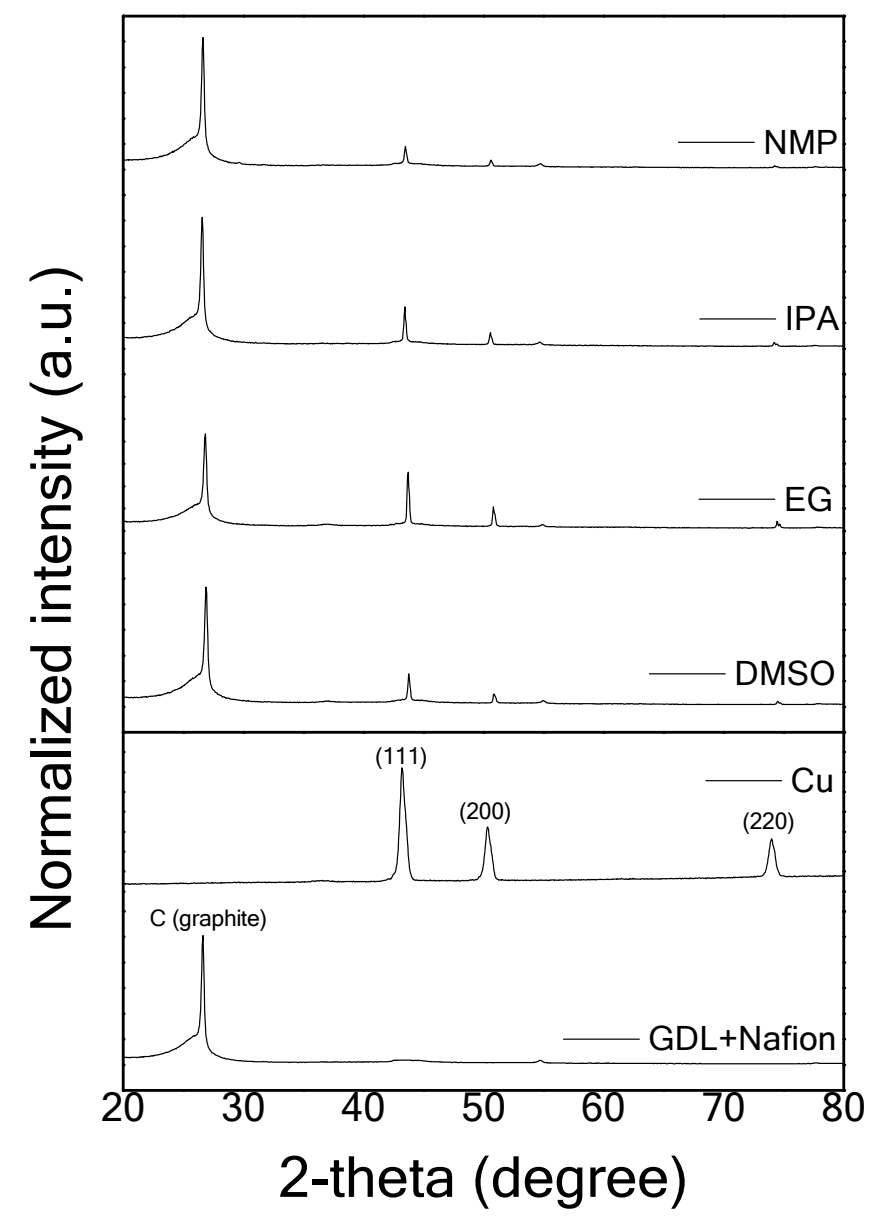

Figure S4. X-ray diffractogram of Cu-GDE samples prepared using several solvents.

S5. Chronoamperometry experiments for all Cu-GDEs during 1 hour catalytic tests.

Figure S5 depicts the chronoamperometry tests (applied constant potential) for one hour experiment. Colored lines indicate the ink-solvent utilized. 


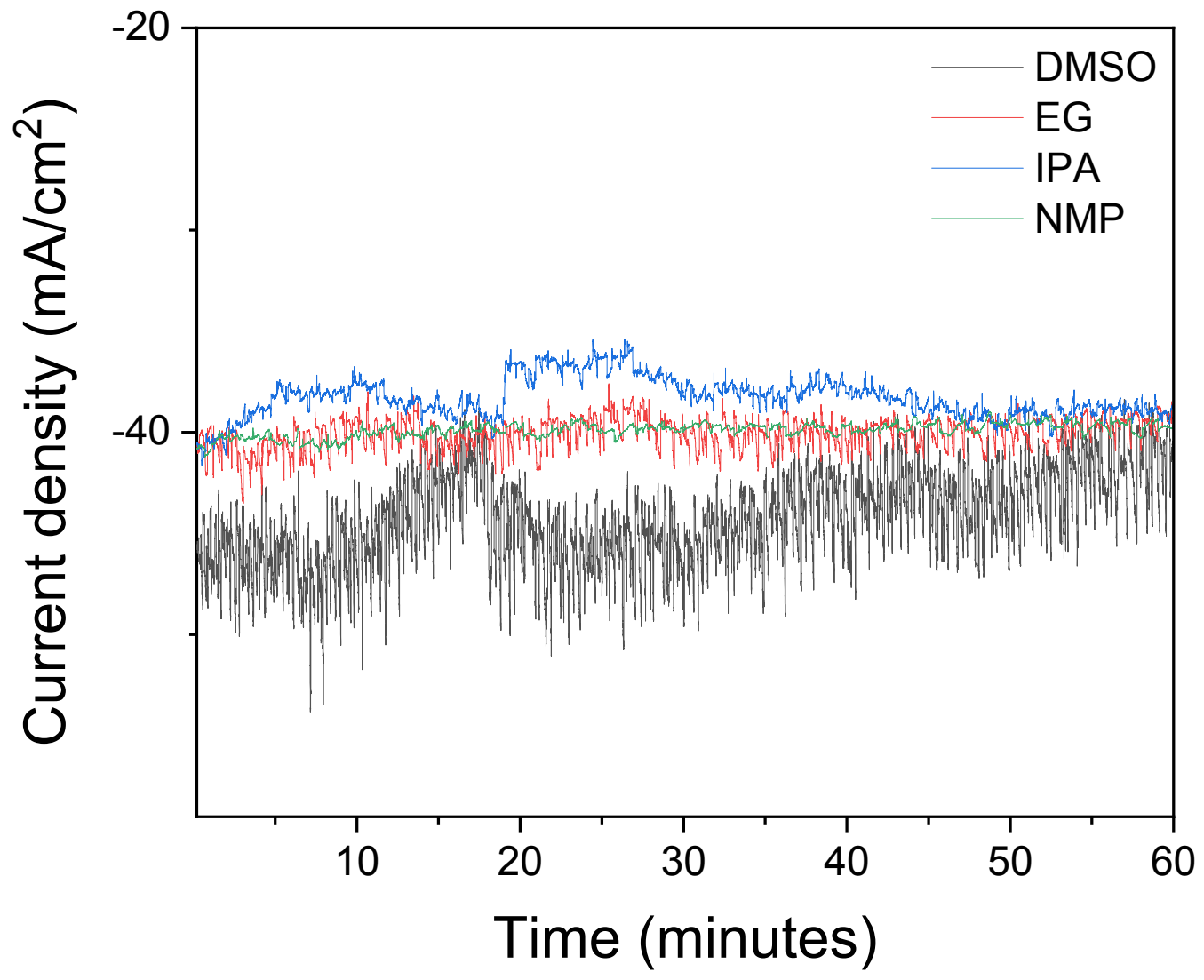

Figure S5. Chronoamperometry experiments of 1 hour duration for all solvent-based catalysts investigated in the present report. 
S6. Overall faradaic efficiency to all products of the electrochemical reduction of $\mathrm{CO}_{2}$.

Figure S6 presents the overall faradaic efficiency to the main reaction products, i.e. $\mathrm{H}_{2}, \mathrm{CO}$,

$\mathrm{CH}_{4}, \mathrm{C}_{2} \mathrm{H}_{4}$ and $\mathrm{C}_{3}$. Formic acid was only identified on high overpotentials but below $1 \%$ faradaic efficiency to all samples. $\mathrm{C}_{3}$ accounts for both propane and propene, since the GC was not able to separate the peaks.
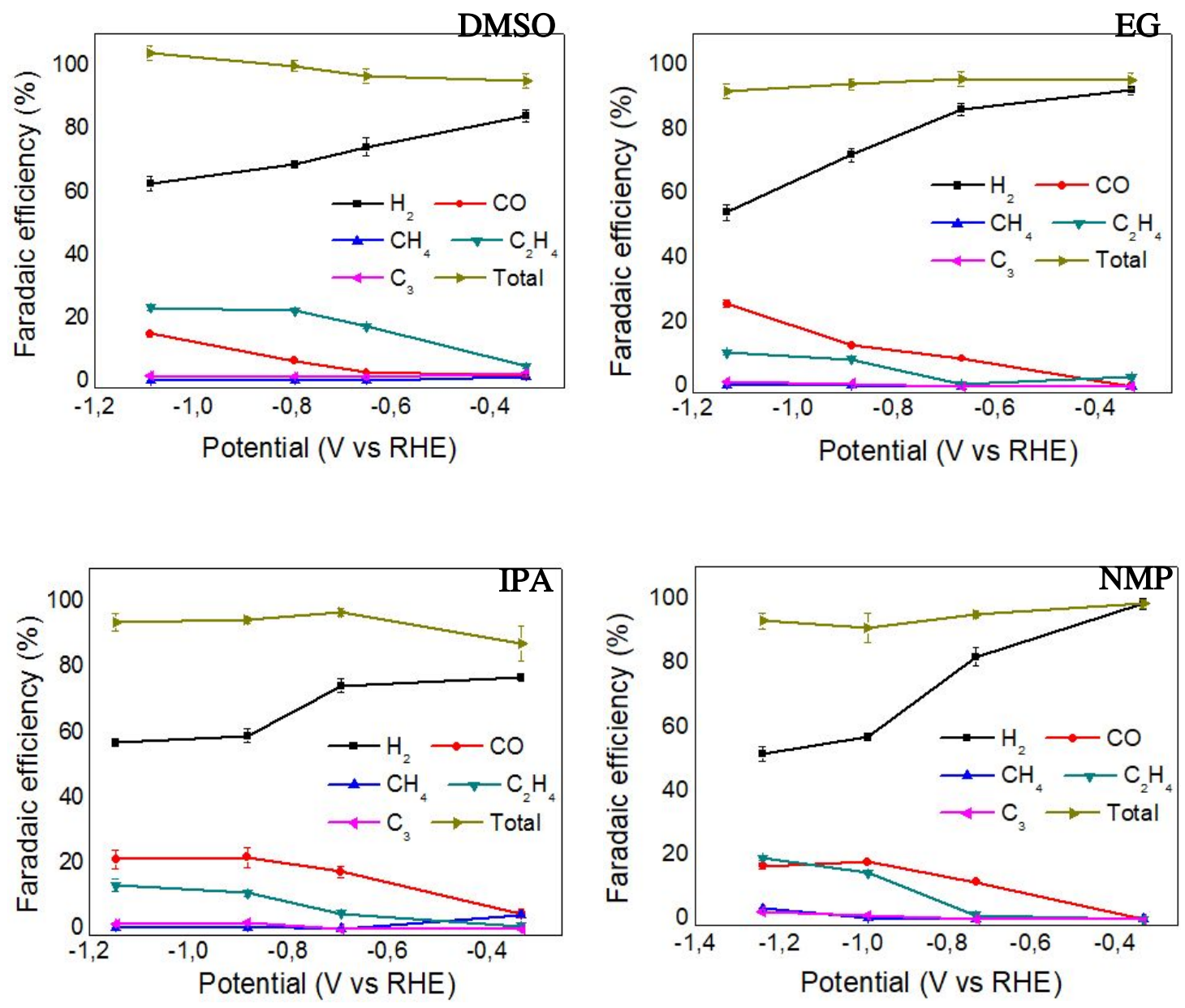
Figure S6. Potential-dependent faradaic efficiency to all $\mathrm{CO}_{2}$ reduction products for $\mathrm{Cu}-\mathrm{GDE}$ samples prepared using different ink-solvents.

S7. Effect of Nafion concentration on carbon monoxide and ethylene faradaic efficiency for DMSO-based GDEs.

Figure $\mathrm{S} 7$ depicts the faradaic efficiency results for $\mathrm{CO}$ and $\mathrm{C}_{2} \mathrm{H}_{4}$ during chronoamperometry tests at $-1.1 \mathrm{~V}$ for $1 \mathrm{~h}$ using $0.3 \mathrm{M} \mathrm{KHCO}_{3}$ as electrolyte for DMSO-based GDEs containing different Nafion concentrations. Higher overall $\mathrm{CO}_{2} \mathrm{RR}$ faradaic efficiency was obtained by $2 \mathrm{wt} \%$ Nafion when compared to $30 \mathrm{wt} \%$ probably due the lower ionomer coverage degree over copper particles, enabling a higher local $\mathrm{pH}$ surrounding the catalyst surface which favored ethylene formation. 


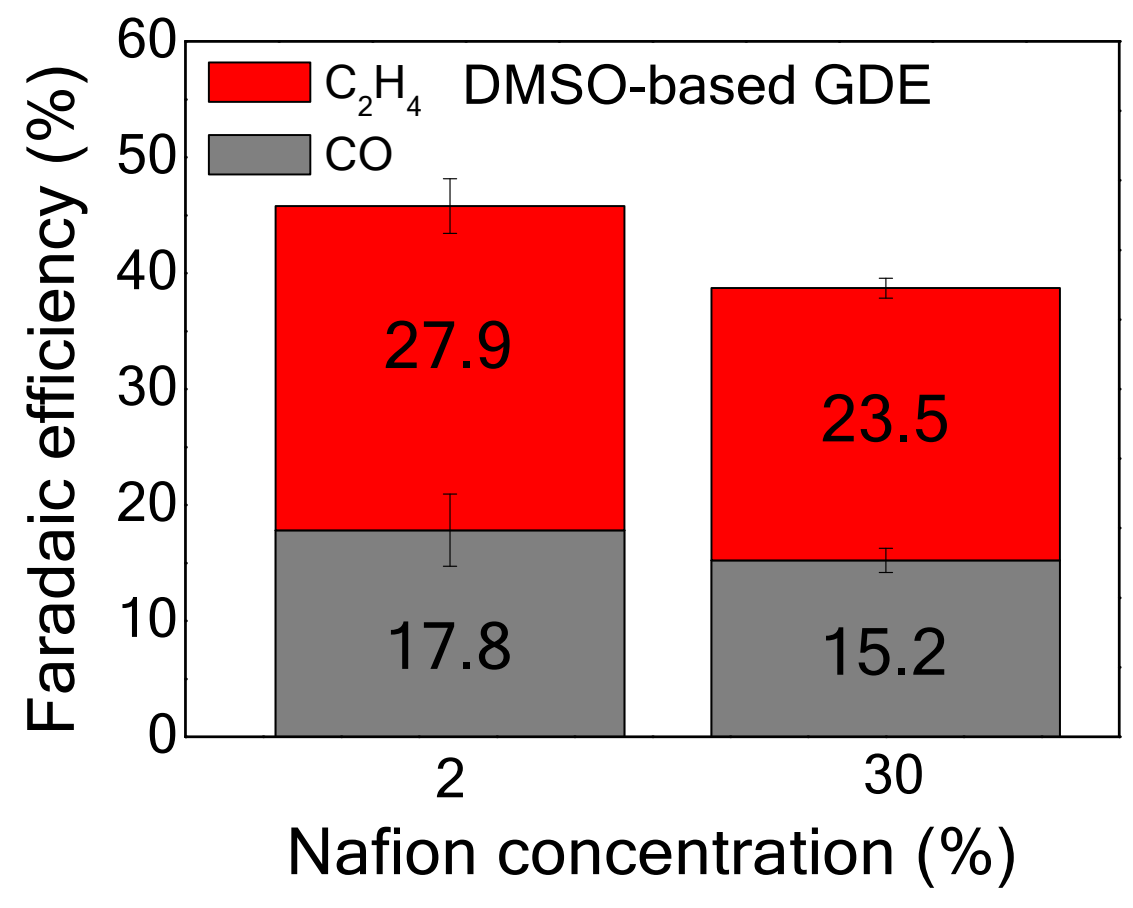

Figure S7. Effect of Nafion concentration on $\mathrm{CO}_{2} \mathrm{RR}$ faradaic efficiency for DMSO-based GDEs. 